\title{
Deep learning neural network seismic big-data analysis of earthquake correlations in distinct seismic regions
}

\author{
Antonios Konstantaras ${ }^{*}$, Nikolaos S. Petrakis ${ }^{2}$, Theofanis Frantzeskakis $^{3}$, Emmanouil \\ Markoulakis $^{3}$, Katerina Kabassi ${ }^{4}$, Ioannis O. Vardiambasis ${ }^{1}$, Theodoros Kapetanakis ${ }^{1}$, Alexandra \\ Moshou $^{1}$ and Emmanuel Maravelakis ${ }^{1}$ \\ Associate Professor, Department of Electronic Engineering, Hellenic Mediterranean University, Romanou 3, \\ Chalepa, GR-73133 Chania, Crete, Greece ${ }^{1}$ \\ Lecturer, Department of Electronic Engineering, Hellenic Mediterranean University, Romanou 3, Chalepa, GR- \\ 73133 Chania, Crete, Greece ${ }^{2}$ \\ Research Scholar, Department of Electronic Engineering, Hellenic Mediterranean University, Romanou 3, Chalepa, \\ GR-73133 Chania, Crete, Greece ${ }^{3}$ \\ Associate Professor and Head, Department of Environment, Ionian University, M. Minotou-Giannopoulou, 29100 \\ Panagoula, Zakynthos, Greece ${ }^{4}$
}

Received: 20-August-2021; Revised: 21-November-2021; Accepted: 23-November-2021

(C)2021 Antonios Konstantaras et al. This is an open access article distributed under the Creative Commons Attribution (CC BY) License, which permits unrestricted use, distribution, and reproduction in any medium, provided the original work is properly cited.

\begin{abstract}
This research work employs deep-learning neural-networks in aiming to unveil the possible existence of a relation between mean rates of seismic activity among consecutive large seismic events and their interim time-intervals. The research is conducted in possibly discrete seismic areas of the steady flow of input strain energy, identified in the southern front of the seismic Hellenic arc. Periods with low-level seismicity in terms of activity result in accumulation of strain energy, which is being stored in under-ground geological faults in the Earth's crust, which in turn are acting as energy storage elements. On the contrary, the occurrence of strong earthquakes acts as a decongestion mechanism which causes the release of significant amounts of the stored energy to the surface of the Earth. Accounting for mean seismicity rates on regular time intervals, e.g., monthly, results in a tool that enables monitoring the underlying management system of the stored seismic energy. The captured information from the processed big-data, regarding not just quantity, but mostly type variation. It was channelled to a deep-learning model whose purpose is the identification and simulation. It was aided by parallel processing training algorithms, of the potential relation of mean seismicity rates, recorded in between consecutive strong earthquakes and their interim time-intervals, in a particular possibly discrete seismic area. Successful training yields a real time dynamic mechanism able to estimate the duration period between the last recorded and next upcoming strong earthquake. The proposed model achieves noteworthy approximations, in the range of approximately two weeks to four months, of the time interval between successive large earthquakes, which reside within the foreshockaftershock time period of each main seismic event. The obtained range falls well-short of the observed mean seismic recurrence times laying in between 1.5 to 2 years.
\end{abstract}

\section{Keywords}

Deep learning, Neural networks applications, Spatio-temporal analysis, Seismicity correlations.

\section{Introduction}

Seismic activity along the Greek vicinity comprises the net product emerging from the critical movement of the tectonic plates beneath the eastern Mediterranean Sea.

\footnotetext{
*Author for correspondence

This work was supported in part by the Institutionalized Laboratory of Computer Technology, Informatics \& Electronic Devices (CompuTech), Chania, Greece under Grant 80280/ELKE/HMU.
}

The latter for the most part is placed on the Eurasian plate, with the Cretan part of it situated in close proximity to the border of the Eurasian tectonic plate with the African tectonic plate [1]. This two-plate convergence gives rise to the southern Hellenic seismic arc, a wide area casting highly unpredictable large seismic activity, that has been a research focal point for multiple reasons, including fundamental seismic research, hydrocarbons exploration and natural hazards safety due to vast tourism inflow [2, 
3]. Long-term prediction of earthquakes aims at estimating their occurrence times years or even decades in advance [4], focusing upon recurrence times $[5,6]$ and various long term earthquake precursors reported in the literature; including, among others, specific changes of known patterns of seismicity manifesting amidst the large earthquakes' preparation stages [7, 8]. Reports of successful cases of long-term earthquake predictions $[9,10]$ have so far been sporadic and in most cases non-repetitive. A number of attempts have been made to support potential long-term earthquake precursors with models of the underlying mechanism [11-13] and inlab confined experiments [14, 15], but these types of attempts present the downfall that they can vary considerably for various different areas $[8,16]$. Some methods $[17,18]$ focused on exploiting the artificialneural-networks' ability to operate as a globalapproximators aiming towards the simulation the behavior of the seismic mechanism. Initial applications show neural networks being employed, alone or in hybrid systems, for mapping input datasets to output datasets, such as detected potential precursors manifesting as long-term seismic pattern changes with the time periods recorded amidst consecutive strong earthquakes in a particular area [19-21].

The proposed method focuses in a potentially distinct seismic region identified as such by [22] covering the southern seismic front of the Hellenic arc and is characterized by nearly steady inflow of strainenergy, attributed to the steady rate of motion of the sub-sinking tectonic-plate of Africa beneath Eurasia, measured at three millimeters on a yearly basis [21]. The steady input flow of energy enables to invoke deep learning neural networks using monthly mean seismicity rates (MMSR) in order to monitor and simulate the strain accumulation and release energy process. It is reported $[6,7]$ that low seismic activity yields in a significant increase in the energy build-up stored in a particular seismogenic area. On the contrary, energy release to the surface, manifesting as actual earthquakes, acts as a decongesting mechanism. Should there be prolonged intervals of minimal recorded seismicity, eventually that shall lead towards the sudden release of vast amounts of, the stored in underground faults, strain energy to the surface, which is depicted as large earthquakes.

Encompassing deep learning neural networks allows for the use of extensive architectures, big training data sets and multiple recursive inputs, all enabled by heterogeneous parallel processing algorithms. The inputs to the deep learning model comprise of rates of mean seismicity that are used to introduce to the latter information regarding strain-energy's capacity accumulated in that particular seismic region. The time-periods amidst consecutive strong earthquakes form the model's required-output. After successful training, the network has the ability to estimate the duration-period amidst the last and next forthcoming large earthquake by constantly assessing the mean rates of the recorded seismicity observed at the possibly seismically distinct southern front of the Hellenic arc.

The results depicted by the deep learning model enhance the observation reported by [22] that this particular area of the southern seismic front of the Hellenic arc behaves as a possible distinct seismic region. Should that prove to be the case, then extending this work to investigate for the possible presence of further neighboring distinct seismic regions could provide a significant means for understanding the seismic clustering phenomenon [23], i.e., whether or not it is possible for a strong earthquake that has occurred in a certain area to trigger the manifestation of a new main strong seismic event in the surrounding region.

\section{Materials and methods}

\subsection{Data pre-processing for homogeneity and} completeness

The Greek Institute of Geodynamics [24] monitors a large part of this region and provides highly accurate seismic data through its forty-five telemetric, digital seismic stations, equipped with broadband 3D component seismometers. The big data extracted are carefully revised, archived and are being made available on the institute's website in multiple chronologically arranged catalogues, in the form of plain text, where each group of data is simply separated by spacing. For the research-needs of this attempt in analyzing seismological big data, the full catalogue has been automatically imported and big data were being extracted, organized and used in such a manner in order to transform them to a suitable format that satisfied the assignment goals. The big data set contains various types of information comprised of more than 290,900 entries, starting from year 1964 and going on as far as 2021, having recorded tremors of magnitude sizes between 0.1 and 7.2 in Richter scale, seismic depths between 0 and 244 in kilometers and precise timestamp in seconds, minutes, hours, day and month the earthquake occurred, as well as the geographic location in latitude (north) and longitude (east), for every 
recorded earthquake. This information is made readily available for each earthquake in the form of a ten-element vector holding the date (year, month, day), the time (hour, minute, second), the location (latitude, longitude and depth), along with the magnitude, in that particular order.

To achieve reliable training of deep learning neural networks, it is important to ensure homogeneity as well as completeness of the biggest seismic to prevent feeding the deep learning neural network with information that is either misleading or false [25]. The data-level of completeness was evaluated by measuring monthly seismicity variations over three decades starting from late $80 \mathrm{~s}$ and onwards where the line of regression demonstrated a notable gradient measured at 0.36 , that falsely indicates an increase in seismic activity throughout the Greek vicinity [21]. Reducing the seismic dataset to measurements of MS > 3.9 indicates that the number of earthquakes with magnitudes equaling or exceeding MS 4.0 appear to be nearly stationary on a yearly basis, whereas their gradient shows a mere slope measured at 0.008 . It is likely that the increase of seismic recording stations and the constant expansion of the seismological network by Greek Institute of Geodynamics enables the recording of additional remote and weaker seismic events that result in the notable rise of weak seismic events' rate.

Further adding to artificially induced effects comes from the installation of modern recorders of seismicity replacing older stations. These are yielding shifts in the magnitudes of the recorded events because of differences among the recorders' characteristics viable for determining the magnitude of each recorded earthquake. Also, fewer artificial effects emerge from the reports of approximate magnitudes in cases where precise measurements of magnitude were not possible [26, 27]. This is made apparent in Figure 1 that displays the distribution of earthquake magnitudes recorded per year. It clearly demonstrated a significant increase in recordings of weaker seismic events with time following the expansion of the seismic recording network and the use of better technologically advanced equipment. Based upon the aforementioned observation that artificially imported changes affect mostly weaker seismic recordings [26], a lower threshold was set at MS 4.0, which was identified using z-statistics [10, 21], to remove a substantial portion of the most affected seismic events from the data to be processed.

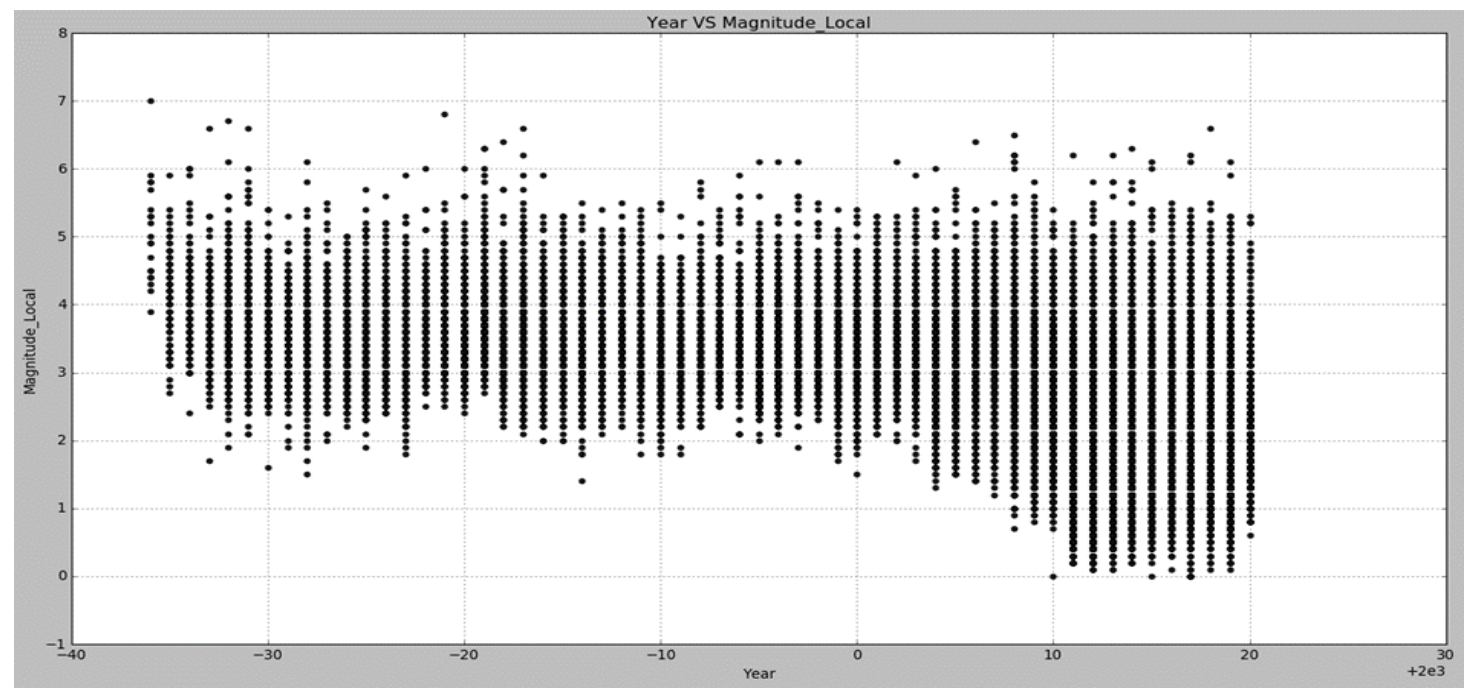

Figure 1 Increased, in number and sensitivity, earthquake recording stations lead to an artificial rise of recorded a number of seismic events attributed solely to the ability to depict significantly weaker earthquakes

Observing the spatial distribution of seismic data allows for early deductions as to where the most intense seismic activity manifests and enables sideby-side observations with additional geographical data like known locations of underground faults. The data by themselves, provide enough information in order to be plotted and examined in threedimensional axis [28, 29] using Cartesian coordinates. Additionally, projected $3 \mathrm{D}$ data can be simultaneously colour-coded to incorporate magnitude as a fourth attribute and be displayed with respect to occurrence times [29], enabling the user to 
observe over certain time periods the seismic activity of a particular seismic area. Such observations are valuable in identifying the initiation of certain underground faults' activity in the region of interest. For that purpose, efforts have been made to spatiotemporally cluster [30] seismic data into potential distinct seismic regions [22] and associate those with known underground faults.

It is difficult to identify the in-depth vertical extent of underground faults, but should a significant number of recorded earthquakes been associated with a particular underground fault, then the hypocentres of those earthquakes give a good indication of the indepth location of the seismically active part of that particular underground fault. Still the longer the depth where the fault is situated the lesser the seismic information that makes it to the surface. This is depicted in Figure 2, which presents recorded earthquake magnitudes with respect to hypocentres' depth location. Figure 2 shows an outer limit narrowing for both smaller and larger seismic events forming a right-arrow shape. It is possible that this effect is caused because the earth's medium. It acts as a dampening mechanism weakens seismic-wave intensity as they propagate to the surface. As a result, larger seismic events are not recorded as strong by surface recording stations, whilst weaker seismic events are getting a lot harder to depict once they reach the Earth's surface. This dampening effect is further enhanced in areas covered by deep sea due to the change from ground to extensive water-medium.

Following the above observations, having noted, data limitations and having compensated for artificial infliction of false information in the recorded data. It was possible to emerge with a complete and homogeneous seismic data set characteristic of the area of interest. This data set was then deployed as the source for potential pattern's extraction by deep learning parallel processing neural networks as described in the next subsection.

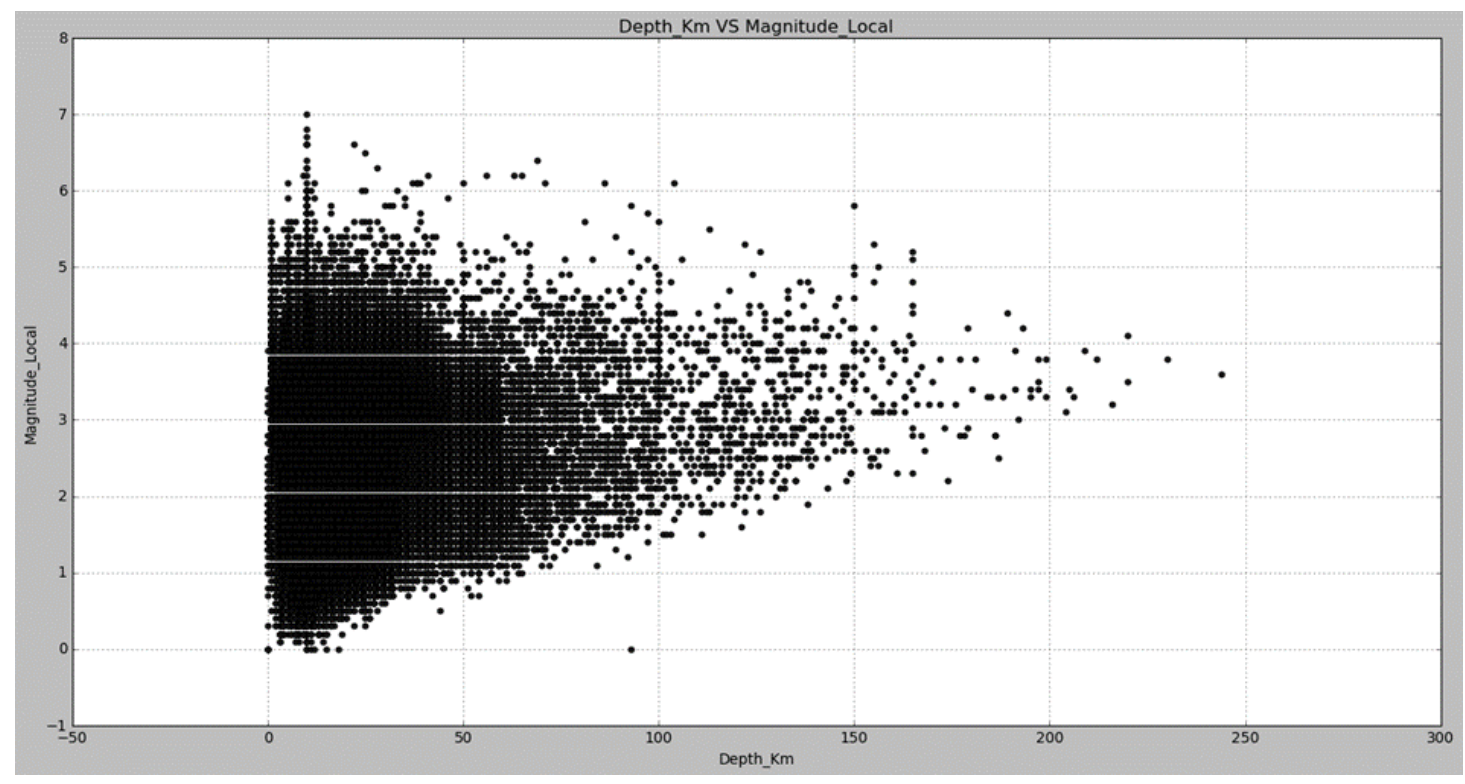

Figure 2 Earthquake magnitude in relation to hypocentral depth

\subsection{Deep learning neural network architecture and training}

Deep learning parallel processing neural networks provide a formidable tool in pattern recognition and features' extraction procedures. The open-source scientific distribution of Anaconda provides a wide variety of tools required to develop and train deep learning artificial neural networks of various architectures and parallel processing training algorithms. The overall goal of the network is to detect whether there is a correlation amongst rates of mean seismicity and duration periods between consecutive large main seismic events, within the possibly seismically distinct southern front of the Hellenic arc [22, 30]. Figure 3 shows the distribution of strong $\mathrm{MS}>=5.0$ main seismic events located throughout the Greek vicinity. 


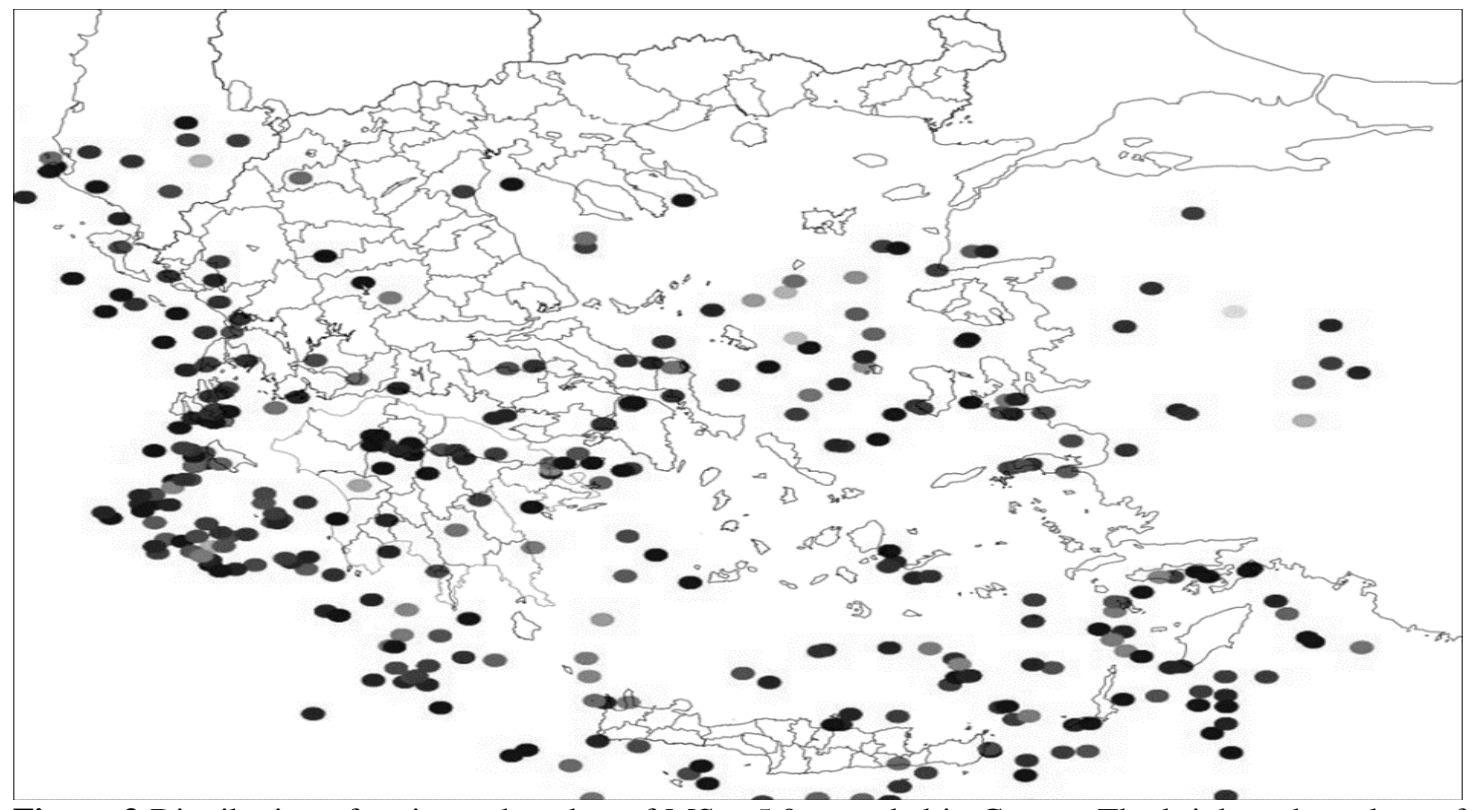

Figure 3 Distribution of main earthquakes of MS $>=5.0$ recorded in Greece. The brighter the colour of the dot, the greater the magnitude of the plotted earthquake

Artificial neural networks $[10,20]$ revolve around perceptron's or neurons, i.e., nodes that receive several inputs, each multiplied by a weight of significance and added to an overall sum, which is then passed through an activation function that produces an output. It is the activation function that decides if a certain node shall activate or not. The inputs of the system consist of external information fed to the system. Each input connects to an inputlayer neuron and these neurons in turn connect to neurons organized in intermediate layers till reaching the final layer of output neurons. A group of structured interconnected neurons is called a neural network. The capabilities of a neural network alternate depending significantly on the extent of its structure, i.e. the number of neurons per hidden-layer as well as the hidden-layers' number. If the system utilizes no hidden layers, it is only capable to represent linearly separable deductions. Any function can be approximated by a neural network with one hidden layer which does contain a continuous mapping from one finite space to another finite space. A neural network with two hidden layers is a system that can represent "an arbitrary decision boundary to arbitrary accuracy with rational activation functions" [31] able to approximate any smooth mapping to any accuracy. With over two layers, the system is able to learn complex representations [32,33].

Each artificial neural network [20] contains features and labels. Features are the data fed into the network used for training purposes. Labels are the values the network is trained upon. These features and labelled values can be of many different types, but the most common types used with artificial neural networks are categorical and numerical data. Categorical data represent a decision between other available choices, like the gender of a species, the month of the year or the handwritten digit a system recognizes. It categorizes it between zero and nine, whilst the problems that have to do with categorical data are called classification problems and usually consist of more than two neurons in the output layer, meaning that the neural network structure has to predict between some cases and operate a classification based on the input data. If the problem is to predict a numerical value, it is called regression problem. The most common example of a regression problem is one where the user predicts the value of an estate based on the number of its rooms and the square feet of the plot.

This research work addresses a regression problem work that aims to dynamically predict the interim duration periods amidst consecutive strong main seismic events, manifesting within a possible distinct seismic zone, given recursively monthly information regarding rates of mean seismicity. Magnitudes that have significant differences in size can be misinterpreted by neural networks, as each neuron uses the sum of these values multiplied by their individual weights and then passes the sum to an 
activation function, hence those values must be normalized before training.

This assignment utilizes the standard score Z-Score method which is expressed by the function (Equation $1)$.

$Z_{i}=\left(X_{i}-\mu\right) / \sigma$

Where $Z_{i}$ is the processed value, $X_{i}$ is the real value, $\mu$ stands for the average value and $\sigma$ for the standard deviation. Every column of data has its own set of $\mu$ and $\sigma$, which are used to reverse this procedure following completion of the deep-learning neural network's operation. Z-Score standardization has been selected over normalization because it is not confined by a bounding range, thus ensuring that even long seismic time-intervals amongst large earthquakes will remain unaffected and won't be cast as outliers [34, 35].

Once data are transformed to similar values the training process is initiated. Training utilizes the TensorFlow and Keras libraries. TensorFlow is a symbolic math library that can also be used for applications in machine learning including deep learning neural networks. It was developed by Google Brain, initially for internal Google use only, and it was then released under the Apache 2.0 license in 2015 [36, 37]. Keras is a Python-based opensource library that supports deep learning neural networks. It is capable of running on top of TensorFlow and it is designed to enable quick experimentation with deep learning neural networks by focusing in being modular, extensible and user friendly. It was developed as a part of the project "Open ended Neuro Electronic Intelligent Robot Operating System (ONEIROS) [38, 39]. Python has distributions of these libraries that the user can encompass in order to produce neural network implementations, of which the Anaconda distribution is used in this work.

Data are being imported either directly from the webcatalogues or from external data files. Depending on the structure and arrangement of the imported data, certain re-arrangements might be needed in order to fit the system requirements, which are being conducted using a data-handling library. The data handling library used in this research work was Pandas. Pandas can handle text data, separated by spaces, new lines, comma separated values, etc., in datasheet files and convert them into data frames, a type of object in Python. The imported data have been organised in columns, each of which corresponding to a different quantity, thus being made readily available for the training process.

Keras documentation, offers a valuable source of information for the user to decide the type of model to be designed and to select a suitable activation function and optimizer for the particular task in hand. This research work employees a sequential model type deep learning neural network with two sets of recursive inputs in the input layer, six hidden layers with one hundred neurons each and a single output layer displayed in Figure 4.

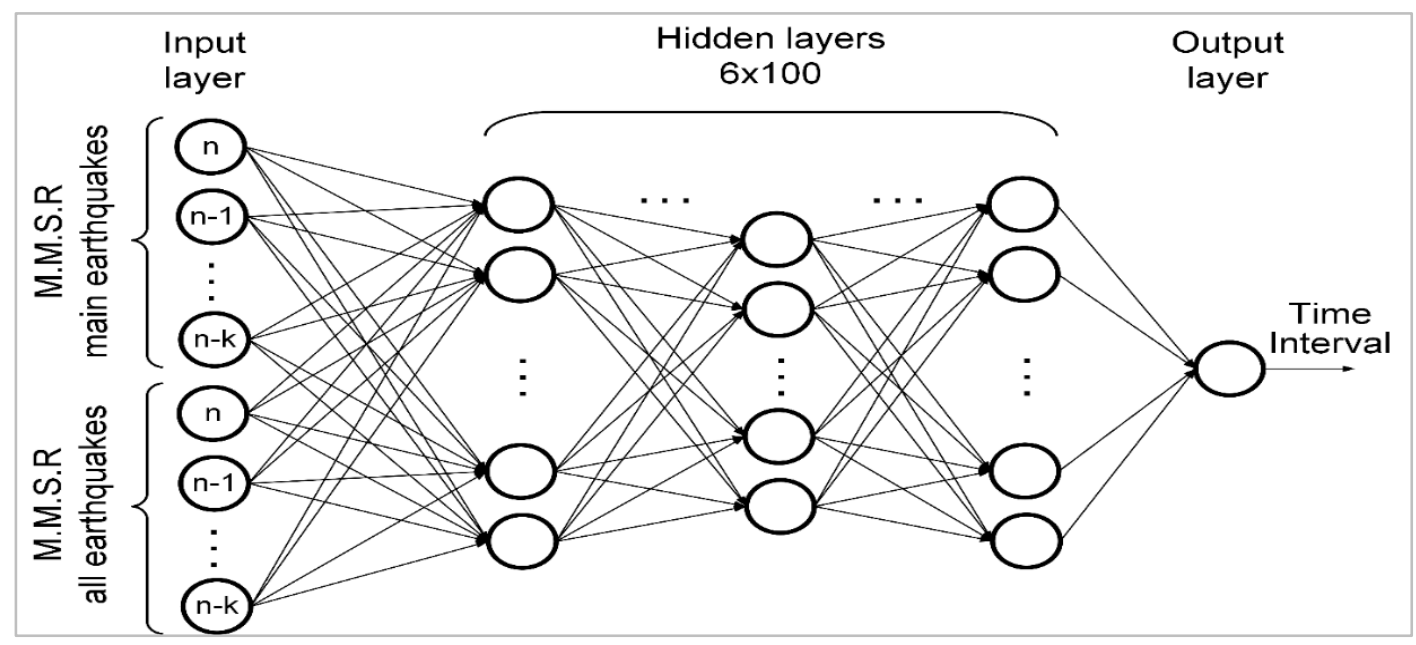

Figure 4 Deep-learning neural network working mechanism architecture 
Every neuron of the system is activated by the rectified linear unit [40], which chooses the maximum between the sum of the weighed imported values and zero. A single crisp output is provided in the output layer as is the case in every regression problem [41-43]. The neural network modifies its weights during the training process, using a supervised learning by exploiting the difference obtained by negating from the neural network's actual crisp output the expected required output already provided by the training dataset. The compilation procedure, i.e. training and testing processes, requires some parameters to be configured by the user, such as the loss method and the optimizer to be used. The loss method is a string parameter that represents a scalar value that the network attempts to minimize during the training of the deep learning neural network model and signifies how close are the predicted values to the true values. Keras provides many loss methods like mean squared error, mean absolute error, categorical cross entropy and many more, described in the Keras losses documentation [38]. The optimizer is a parameter that defines learning sizes such as learning rate, momentum, decay of learning rate and more, depending on the optimizer used. There are many different and ready to use optimizers that Keras supports, such as Stochastic gradient descent, Adam, Adamax and more, but the user is free to self-manage the optimizers parameters should that be deemed preferable. For this particular research work network's best performance was obtained by incorporating as loss-parameter the mean-squared-error along with an optimizer of type Adam.

Training is initiated by applying the Fit method upon the model. The Fit method's arguments are the input data or features, i.e. the data the network is trained upon, a batch size, an epoch size and a validation dataset. The validation dataset is a slice of the overall dataset, approximately about thirty percent of the overall dataset, kept unknown during the training process to the deep learning neural network. Instead, it is used after training to validate how well the model handles unknown data, a process necessary to prevent overtraining the neural network that causes data-overfitting [44-47]. Information gain does not work well for attributes with large number of distinct values [48] because of overfitting as is the case with the task at hand. To address this problem, a dropout rate of 0.5 [49] was incorporated to randomly set input units to zero at that specific frequency rate, at each step during training. The remaining inputs that are not being set to zero are scaled up by $1 /(1$ - rate $)$ so as for the sum over all inputs to remain unchanged. Dropout only applies during training and as such no values are being dropped during inference. The training results are stored in a type-history variable that keeps a history record of the models' attributes like training loss, successive epochs' metric values, values for validation loss and validation metrics. The normalized output of the trained model is saved to an external file for later use holding custom user data, in the form of a spreadsheet file. It is important to maintain the means and standard deviation of the output values, so as the Z-Score method can be used to extract the real values depicted by the network from the normalized results.

\section{Results}

MMSR is the independent variables that were used as inputs to the deep learning neural network model. It enables the latter to monitor the mechanism of energy storage and release processes of the potentially seismically distinct southern front of the seismic Hellenic arc. The range is between $35.20^{\circ}$ to $37.50^{\circ}$ in latitude and $20.50^{\circ}$ to $27.50^{\circ}$ in longitude as has been indicated by $[22,50]$. The inputs cover current, and previous, thereby introducing recursive information, rates of mean seismicity for main seismic events and for those of foreshocks and aftershocks. These are calculated from the respective cumulative activity curves using the regression line fitting, so as the model can potentially deduce patterns, not just by the released by all earthquake's overall energy but also from the energy released solely by the main earthquakes.

The aforementioned information along with measurements of the duration period amongst consecutive large earthquakes, measuring magnitudes exceeding a certain user-defined value of $\mathrm{MS} \geq 6.0$ $[51,52]$, as the required output, form the training dataset applied to the deep-learning neural network via a parallel processing training algorithm, as was discussed in section 2. The duration intervals inbetween the last and the immediate upcoming strong earthquake are the dependent variable forming the deep learning neural network's required output. These time-intervals used for training were obtained by converting the occurrence datetime of successive large earthquakes into a unique number using UNIX timestamp and then derive their difference.

The loss progress function of the number of training epochs using training and validation data is being displayed in Figure 5. Although training was conducted over five thousand training epochs the loss 
function indicates a variation between training and validation error at around two hundred training epochs. This is attributed to overtraining the deep learning neural causing it to memorize rather than understand the behaviour of the processed timeseries, which affects its operation when presented with unseen during training data.

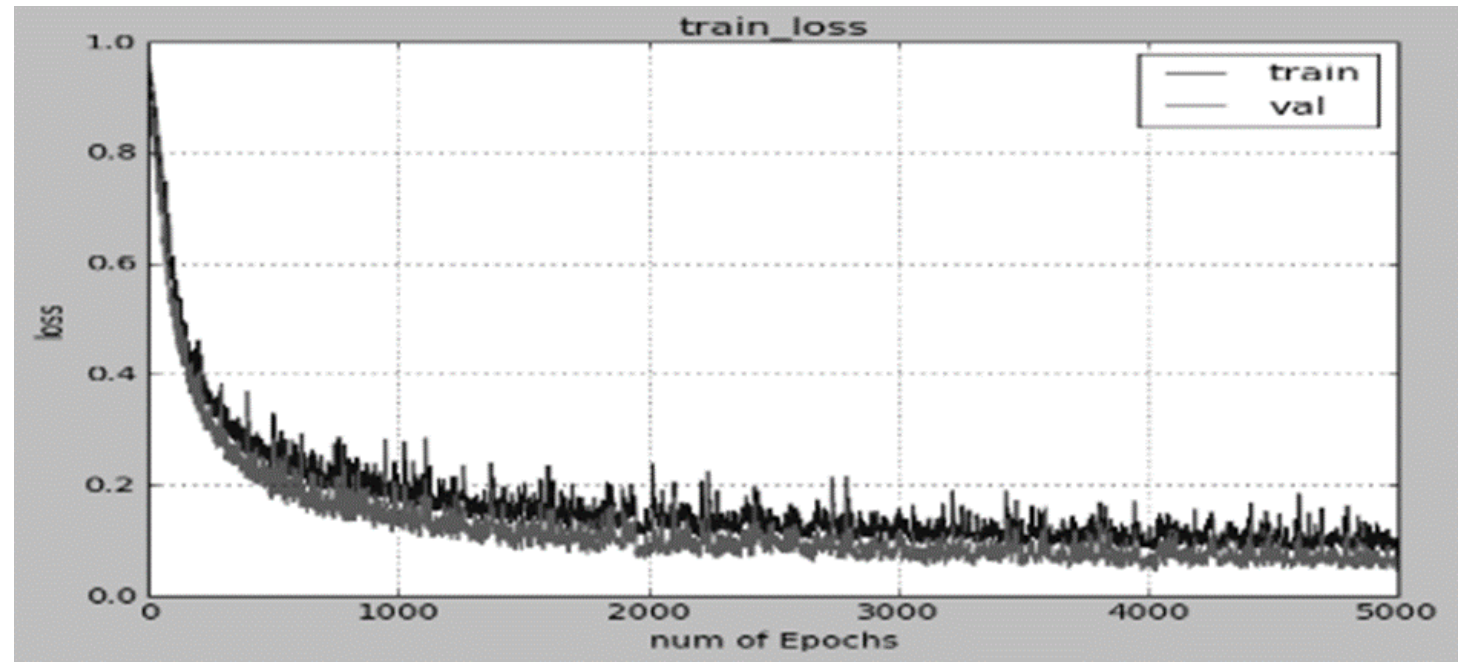

Figure 5 Deep learning neural network training (brighter line) and validation (darker line) loss progress minimization over the number of training epochs

By encompassing validation data during the training process and cataloguing the specific parameters of the deep learning neural network model for every training epoch enables to user to derive the optimum number of epochs at which the current parameters of the model yield the best possible performance when presented with unknown data from the same seismic region. The crisp output of the model provides a number that corresponds to the difference in time amongst the last occurred and the next expected strong earthquake. This number when added to the respective number corresponding to the date and time of the last strong earthquake pinpoints the estimated datetime of the potential occurrence of the immediate upcoming strong earthquake in the seismically active region under investigation as shown in Table 1.

Table 1 List of all main seismic events, kept unknown to the deep-learning neural network, with magnitudes of MS $\geq 5.5$ within the potentially seismically distinct southern front of the Hellenic arc from 2009 till 2021, along with the depicted estimations of the date of occurrence

\begin{tabular}{|c|c|c|c|c|c|c|c|c|c|c|}
\hline Year & Month & Day & Hour & Min & Sec & Lat & Long & Depth & Mag & $\begin{array}{lll}\text { Estimated } & \text { Date } \\
\text { Comments } & & \\
\end{array}$ \\
\hline 2021 & JUN & 21 & 22 & 14 & 15 & 36.38 & 27.07 & 15 & 5.7 & \multirow[b]{2}{*}{$\begin{array}{l}06 / 07 / 2021 \\
\text { (The seismic energy } \\
\text { released by two } \\
\text { significant EQs rather } \\
\text { than a larger single EQ) }\end{array}$} \\
\hline 2021 & AUG & 01 & 04 & 31 & 26 & 36.38 & 27.08 & 16 & 5.4 & \\
\hline 2019 & NOV & 27 & 7 & 23 & 42.7 & 35.69 & 23.26 & 71 & 6.1 & $02 / 02 / 2020$ \\
\hline 2018 & OCT & 30 & 15 & 12 & 2.0 & 37.46 & 20.45 & 6 & 5.5 & \multirow{2}{*}{$\begin{array}{l}18 / 6 / 2018 \\
\text { (the MS 5.5 EQ was } \\
\text { either triggered by or is } \\
\text { an aftershock of the MS } \\
6.6 \text { EQ) }\end{array}$} \\
\hline 2018 & OCT & 25 & 22 & 54 & 49.6 & 37.34 & 20.51 & 10 & 6.6 & \\
\hline 2017 & JUL & 20 & 22 & 31 & 11.7 & 36.96 & 27.43 & 10 & 6.2 & $30 / 04 / 2017$ \\
\hline 2015 & APR & 16 & 18 & 7 & 44.9 & 35.23 & 26.82 & 37 & 6.1 & \multirow{3}{*}{$\begin{array}{l}27 / 12 / 2014 \\
(25 / 5 / 2015 \text { when } \\
\text { retrained with data until } \\
\text { AUG-2014) }\end{array}$} \\
\hline 2014 & AUG & 29 & 3 & 45 & 5.6 & 36.67 & 23.67 & 97 & 5.7 & \\
\hline 2014 & APR & 4 & 20 & 8 & 7.4 & 37.20 & 23.73 & 113 & 5.5 & \\
\hline 2013 & OCT & 12 & 13 & 11 & 53.0 & 35.50 & 23.28 & 65 & 6.2 & $06 / 11 / 2013$ \\
\hline 2011 & APR & 1 & 13 & 29 & 10.5 & 35.64 & 26.56 & 63 & 6.2 & $21 / 03 / 2011$ \\
\hline
\end{tabular}


Antonios Konstantaras et al.

\begin{tabular}{|c|c|c|c|c|c|c|c|c|c|c|}
\hline & & & & & & & & & & $22 / 10 / 2009$ \\
\hline 2009 & NOV & 3 & 5 & 25 & 9.3 & 37.39 & 20.35 & 39 & 5.6 & (The seismic energy \\
\hline 2009 & FEB & 16 & 23 & 16 & 38.5 & 37.13 & 20.78 & 15 & 5.5 & $\begin{array}{l}\text { released by two } \\
\text { significant EQs rather } \\
\text { than a larger single } E Q \text { ) }\end{array}$ \\
\hline 2008 & FEB & 14 & 10 & $\underline{9}$ & 23.4 & 36.50 & 21.78 & $\underline{41}$ & 6.2 & N/A - used for training \\
\hline
\end{tabular}

\section{Discussion}

The time periods from October 1964 to July 2008, April 20011, October 2013, May 2014, April 2015, July 2017, October 2018, November 2019, respectively, have been encompassed to form consecutive training datasets within the potentially distinct seismic region reported by [22], ranging between $35.2^{\circ}-37.5^{\circ} \mathrm{N}$ and $20.5^{\circ}-27.5^{\circ}$ E. During the time-interval between 2009 and 2019 six earthquakes with magnitudes $\mathrm{MS} \geq 6.0$ have occurred measuring a meantime of reoccurrence of just over 1.5 years. For ease of reference in the observations to follow, Table 1 presents all the main seismic events, unknown to the deep learning neural network, of MS $\geq 5.5$ in the possibly distinct seismic region of interest from 2009 till 2019. The obtained results demonstrate specificity at 0.833 , negative predictive value at 0.833 and positive predictive value at 0.857 [53], based upon the estimated and actual occurrence times of large seismic events.

The first training dataset (1964-2008) provides the deep learning neural network with information regarding rates of mean seismicity along with duration periods amongst subsequent strong earthquakes till the 20th of February, 2008 MS 6.0 earthquake. Once the training procedure concluded, the network received recursive input information regarding solely rates of mean seismicity corresponding to interim duration periods in between past main seismic events of magnitude $\mathrm{MS} \geq 6.0$. At that point the network tries to derive the duration period to the immediate upcoming large earthquake, unknown to it as it falls outside of the training data set used. The network's output was initially measured at 53218242. Adding that to the last large earthquake's datetime of occurrence, i.e. February 20th, 2008, corresponded to the calendar date at October 22nd, 2009. Though this result appears to stray substantially with respect to the actual daytime of the immediate consecutive $\mathrm{MS} \geq 6.0$ large earthquake (April 1st, 2011 displaying a magnitude of MS 6.2) within the aforementioned potentially distinct seismic region. A more careful examination of the seismic activity in the area shows that the accumulated stress energy of the expected earthquake was actually released by two significantly large seismic events with magnitudes recorded in MS 5.5 1418 and MS 5.6 on the 16th of February 2009 and 3rd of November 2009, respectively.

Following that observation, the deep learning neural network was retrained with information regarding rates of mean seismicity along with duration periods amongst subsequent strong earthquakes till the November 3rd, 2009, to compensate for the released seismic energy. Once the training procedure concluded, the network received recursive input information regarding solely rates of mean seismicity corresponding to interim duration periods in between past main seismic events of magnitude $\mathrm{MS} \geq 6.0$. At that point the network tries to derive the duration period to the immediate upcoming large earthquake, unknown to it as it falls outside of the training data set used. The network's output was initially measured at 42076874. Adding that to the last large earthquake's datetime of occurrence, i.e. November 3rd, 2009, corresponded to the calendar date at March 21st, 2011, a mere eleven days earlier than the actual recorded MS 6.2 earthquake on April 1st, 2011.

The deep learning neural network was then retrained with information regarding rates of mean seismicity along with duration periods amongst subsequent strong earthquakes till the April 1st, 2011, to compensate for the released seismic energy. Once the training procedure concluded, the network received recursive input information regarding solely rates of mean seismicity corresponding to interim duration periods in between past main seismic events of magnitude $\mathrm{MS} \geq 6.0$. At that point the network tries to derive the duration period to the immediate upcoming large earthquake, unknown to it as it falls outside of the training data set used. The network's output was initially measured at 82107871. Adding that to the last large earthquake's datetime of occurrence, i.e., April 1st, 2011, corresponded to the calendar date at November 6th, 2013, approximately three weeks later than the actual recorded earthquake of MS 6.2 on October 12th, 2013.

Next, the deep learning neural network was retrained with information regarding rates of mean seismicity along with duration periods amongst subsequent strong earthquakes till the October 12th, 2013, to 
compensate for the released seismic energy. Once the training procedure concluded, the network received recursive input information regarding solely rates of mean seismicity corresponding to interim duration periods in between past main seismic events of magnitude $\mathrm{MS} \geq 6.0$. At that point the network tries to derive the duration period to the immediate upcoming large earthquake, unknown to it as it falls outside of the training data set used. The network's output was initially measured at 38114447 . Adding that to the last large earthquake's datetime of occurrence, i.e. October 12th, 2013, corresponded to the calendar date at December 27th, 2014. The actual large earthquake occurred just short of four months later, on the April 16th, 2015. The nearly four-month latency in occurrence might be due to the narrowing of the cumulative stress energy by two smaller earthquakes of magnitudes of MS 5.5 and MS 5.7 that occurred in the vicinity of the potentially distinct seismic region on April 4th, 2014 and August 29th, 2014, respectively.

Following that observation, the deep learning neural network was retrained with information regarding rates of mean seismicity along with duration periods amongst subsequent strong earthquakes till the August 29th, 2014, to compensate for the released seismic energy. Once the training procedure concluded, the network received recursive input information regarding solely rates of mean seismicity corresponding to interim duration periods in between past main seismic events of magnitude $\mathrm{MS} \geq 6.0$. At that point the network tries to derive the duration period to the immediate upcoming large earthquake, unknown to it as it falls outside of the training data set used. The network's output was initially measured at 23199572. Adding that to the last large earthquake's datetime of occurrence, i.e., August 29th, 2014, corresponded to the calendar date at May 25th, 2015, approximately a month and a half later than the actual recorded earthquake of MS 6.1 on April 16th, 2015.

The deep learning neural network was then retrained with information regarding rates of mean seismicity along with duration periods amongst subsequent strong earthquakes till the April 16th, 2015, to compensate for the released seismic energy. Once the training procedure concluded, the network received recursive input information regarding solely rates of mean seismicity corresponding to interim duration periods in between past main seismic events of magnitude $\mathrm{MS} \geq 6.0$. At that point the network tries to derive the duration period to the immediate upcoming large earthquake, unknown to it as it falls outside of the training data set used. The network's output was initially measured at 64350234 . Adding that to the last large earthquake's datetime of occurrence, i.e. April 16th, 2015, corresponded to the calendar date at April 30th, 2017, approximately three and half months earlier than the actual recorded earthquake of MS 6.2 on July 20th, 2017.

Moving on, the deep learning neural network was then retrained with information regarding rates of mean seismicity along with duration periods amongst subsequent strong earthquakes till the July 20th, 2017, to compensate for the released seismic energy. Once the training procedure concluded, the network received recursive input information regarding solely rates of mean seismicity corresponding to interim duration periods in between past main seismic events of magnitude $\mathrm{MS} \geq 6.0$. At that point the network tries to derive the duration period to the immediate upcoming large earthquake, unknown to it as it falls outside of the training data set used. The network's output was initially measured at 26055940 . Adding that to the last large earthquake's datetime of occurrence, i.e. July 20th, 2017, corresponded to the calendar date at June 18th, 2018, close to four months earlier than the actual recorded earthquake of MS 6.6 on October 25th, 2018. This earthquake was accompanied by yet another large earthquake of MS 5.5 on October 30th, 2018, in the nearby vicinity that might either be an aftershock of the MS 6.6 earthquake or another main earthquake triggered by the latter.

Second last, the deep learning neural network was then retrained with information regarding rates of mean seismicity along with duration periods amongst subsequent strong earthquakes till the October 30th, 2018, to compensate for the released seismic energy. Once the training procedure concluded, the network received recursive input information regarding solely rates of mean seismicity corresponding to interim duration periods in between past main seismic events of magnitude $\mathrm{MS} \geq 6.0$. At that point the network tries to derive the duration period to the immediate upcoming large earthquake, unknown to it as it falls outside of the training data set used. The network's output was initially measured at 40176089 . Adding that to the last large earthquake's datetime of occurrence, i.e. October 30th, 2018, corresponded to the calendar date at February 2nd, 2020, just over two months later than the actual recorded earthquake of MS 6.1 on November 27th, 2019. 
Lastly, the deep learning neural network was then retrained with information regarding rates of mean seismicity along with duration periods amongst subsequent strong earthquakes till the November 27th, 2019, to compensate for the released seismic energy. Once the training procedure concluded, the network received recursive input information regarding solely rates of mean seismicity corresponding to interim duration periods in between past main seismic events of magnitude $\mathrm{MS} \geq 6.0$. At that point the network tries to derive the duration period to the immediate upcoming large earthquake, unknown to it as it falls outside of the training data set used. The network's output was initially measured at 50712147. Adding that to the last large earthquake's datetime of occurrence, i.e. November 27th, 2019, corresponded to the calendar date at July 6th, 2021. Careful examination of the seismic activity in the area shows that the accumulated stress energy of the expected earthquake was actually released by two significantly large earthquakes, of magnitudes MS 5.7 and MS 5.4 on June 15th, 2021 and August 8th, 2021, respectively, with the latter having been recorded as an aftershock of the former; and the expected date of occurrence falls within that time interval.

Overall, the deep learning neural network appears to have depicted the behavior of the possible underlying distinct seismic region. In effect, the deep learning neural network estimates the time interval between successive large earthquakes within the temporal sphere of influence [54, 55] of the next forthcoming large main earthquake. Also, in the cases where the stored seismic energy was released by a number of medium-large sized earthquakes, instead of a single strong earthquake, the deep learning neural network's estimated time of occurrence did fall in all cases within the time-window amidst the actual manifestation of those earthquakes. A complete list of abbreviations is shown in Appendix I.

\section{Conclusion and future work}

This method, though, is not an attempt towards earthquake prediction. Instead, it aims to estimate when a specific potentially distinct seismic area has gained enough strain energy that could result in a large earthquake. In spite the fact that energy accumulation appears to be fairly constant mainly due to the steady motion of the African plate moving beneath the Eurasian plate by approximately $3 \mathrm{~mm}$ per year, the user needs to carefully monitor significant releases of energy from seismic events close to the threshold magnitude selected as the minimum reference magnitude in order to enable us to estimate time intervals between large earthquakes. As reported earlier, in those cases of energy release by lesser yet significant seismic events, the deep learning neural networks needs to be retrained to encompass that information and adapt accordingly thereby revising the estimated time period to next subsequent large earthquake. The results presented appear to closely approximate the time interval between the last and the next upcoming consecutive large earthquake deviating only in the range of a few months and in some cases even less; residing within the foreshock-aftershock time period of each main seismic event. These results enhance the observation reported by [22] that this particular area of the southern Hellenic seismic arc behaves as a distinct seismic region.

Future research by the authors shall focus upon minimizing the architecture of the deep learning neural network, narrowing it down to the neurons that appear to make a significant contribution to the actual output of the network. It also validates the effect this has to the results with respect to the expected outputs. Also, this research work shall be extended to cover other potentially distinct seismic regions in the Greek vicinity, such as those in the Ionian Sea and the Cyclades, areas of significant interest in terms of tourism and hydrocarbon exploration. Should any of these areas also be casted as potential distinct seismic regions then it might be possible to deploy deep learning to investigate for the presence of seismic clustering phenomenon [23], i.e., whether a sizeable earthquake in one area can trigger another main earthquake in the surrounding vicinity.

\section{Acknowledgment}

This research would not have been possible without the efforts of the multiple scientists of the Geological Institute of the National Observatory of Athens (GI-NOA) that have made readily and freely available the aforementioned seismological data.

\section{Conflicts of interest}

The authors have no conflicts of interest to declare.

\section{References}

[1] Dilek Y. Collision tectonics of the Mediterranean region: causes and consequences. Special PapersGeological Society of America. 2006.

[2] Gao H, Tong X, Wen Z, Wang Z. The tectonic evolution of the eastern Mediterranean basin and its control on hydrocarbon distribution. Journal of Petroleum Science and Engineering. 2019; 178:389407. 
[3] Kuribayashi E. Lifeline earthquake hazard zonation in socio-economic aspects. Lifeline Systems. 1991.

[4] Hayakawa M. Earthquake prediction with radio techniques. John Wiley \& Sons; 2015; 1-17.

[5] Nomura S, Ogata Y, Nadeau RM. Space time model for repeating earthquakes and analysis of recurrence intervals on the San Andreas Fault near Parkfield, California. Journal of Geophysical Research: Solid Earth. 2014; 119(9):7092-122.

[6] González Á, Serra I, Corral Á. The global statistical distribution of time intervals between consecutive earthquakes. In EGU general assembly conference abstracts 2020 (p. 13309).

[7] Papadimitriou P. Identification of seismic precursors before large earthquakes: decelerating and accelerating seismic patterns. Journal of Geophysical Research: Solid Earth. 2008; 113(B04):1-19.

[8] Chiao LY, Kuo BY. Multiscale seismic tomography. Geophysical Journal International. 2001; 145(2):51727.

[9] Wyss M, Burford RO. Occurrence of a predicted earthquake on the San Andreas fault. Nature. 1987; 329(6137):323-5.

[10] Bodri B. A neural-network model for earthquake occurrence. Journal of Geodynamics. 2001; 32(3):289310.

[11] Eneva M, Benzoin Y. Application of pattern recognition techniques to earthquake catalogs generated by model of segmented fault systems in three dimensional elastic solids. Journal of Geophysical Research: Solid Earth. 1997; 102(B11):24513-28.

[12] Meyers RA. Encyclopedia of complexity and systems science. New York: Springer; 2009.

[13] Falcone G, Spassiani I, Ashkenazy Y, Shapira A, Hofstetter R, Havlin S, et al. An operational earthquake forecasting experiment for israel: preliminary results. Frontiers in Earth Science. 2021; 9:1-10.

[14] Pu Y, Chen J, Apel DB. Deep and confident prediction for a laboratory earthquake. Neural Computing and Applications. 2021; 33:1691-701.

[15] Shokouhi P, Girkar V, Riviere J, Shreedharan S, Marone C, Giles CL, et al. Deep learning can predict laboratory quakes from active source seismic data. Geophysical Research Letters. 2021; 48(12).

[16] Triep EG, Sykes LR. Frequency of occurrence of moderate to great earthquakes in intracontinental regions: implications for changes in stress, earthquake prediction, and hazards assessments. Journal of Geophysical Research: Solid Earth. 1997; 102(B5):9923-48.

[17] Adeli H, Panakkat A. A probabilistic neural network for earthquake magnitude prediction. Neural Networks. 2009; 22(7):1018-24.

[18] Asencio-cortés G, Martínez-alvarez F, Troncoso A, Morales-esteban A. Medium-large earthquake magnitude prediction in Tokyo with artificial neural networks. Neural Computing and Applications. 2017; 28(5):1043-55.
[19] Lakkos S, Hadjiprocopis A, Comley R, Smith P. A neural network scheme for earthquake prediction based on the seismic electric signals. In proceedings of IEEE workshop on neural networks for signal processing 1994 (pp. 681-9). IEEE.

[20] Yin F, Wang J, Guo C. Advances in neural networks. In international symposium on neural networks, Dalian, China. 2004.

[21] Konstantaras A, Vallianatos F, Varley MR, Makris JP. Soft-computing modelling of seismicity in the southern Hellenic arc. IEEE Geoscience and Remote Sensing Letters. 2008; 5(3):323-7.

[22] Konstantaras AJ. Expert knowledge-based algorithm for the dynamic discrimination of interactive natural clusters. Earth Science Informatics. 2016; 9(1):95-100.

[23] Bressan G, Barnaba C, Peresan A, Rossi G. Anatomy of seismicity clustering from parametric space-time analysis. Physics of the Earth and Planetary Interiors. 2021.

[24] http://www.gein.noa.gr/en. Accessed 18 August 2021.

[25] Haeffele BD, Vidal R. Global optimality in neural network training. In proceedings of the conference on computer vision and pattern recognition 2017 (pp. 7331-9).

[26] Leptokaropoulos KM, Papadimitriou EE, Orleckasikora B, Karakostas VG. Seismicity rate changes in association with the evolution of the stress field in northern Aegean Sea, Greece. Geophysical Journal International. 2012; 188(3):1322-38.

[27] Shapiro S. Fluid-induced seismicity. Cambridge University Press; 2015.

[28] Axaridou A, Chrysakis I, Georgis C, Theodoridou M, Doerr M, Konstantaras A, et al. 3D-SYSTEK: recording and exploiting the production workflow of 3D-models in Cultural Heritage. In the 5th international conference on information, intelligence, systems and applications 2014 (pp. 51-6). IEEE.

[29] Maravelakis E, Konstantaras A, Kabassi K, Chrysakis I, Georgis C, Axaridou A. 3DSYSTEK web-based point cloud viewer. In the 5 th international conference on information, intelligence, systems and applications 2014 (pp. 262-6). IEEE.

[30] Konstantaras AJ, Katsifarakis E, Maravelakis E, Skounakis E, Kokkinos E, Karapidakis E. Intelligent spatial-clustering of seismicity in the vicinity of the Hellenic seismic arc. Earth Science Research. 2012; $1(2): 1-10$.

[31] Wang J, Yi Z, Zurada JM, Lu BL, Yin H. Advances in neural networks. In third international symposium on neural networks 2006. Springer Nature.

[32] Shaw BE, Carlson JM, Langer JS. Patterns of seismic activity preceding large earthquakes. Journal of Geophysical Research: Solid Earth. 1992; 97(B1):47988.

[33] Sergaki E, Spiliotis G, Vardiambasis IO, Kapetanakis T, Krasoudakis A, Giakos GC, et al. Application of ANN and ANFIS for detection of brain tumors in MRIs by using DWT and GLCM texture analysis. In international conference on imaging systems and techniques 2018 (pp. 1-6). IEEE. 
[34] Urdan TC. Statistics in plain English. Routledge; 2016.

[35] Pereira FC, Borysov SS. Machine learning fundamentals. In mobility patterns, big data and transport analytics 2019 (pp. 9-29). Elsevier.

[36] James AP. Deep learning classifiers with memristive networks: theory and applications. Springer; 2019.

[37] Lee H. Foundations of applied statistical methods. Heidelberg: Springer; 2014.

[38] https://github.com/fchollet/keras. Accessed 18 August 2021.

[39] https://www.manning.com/books/deep-learning-withr. Accessed 18 August 2021.

[40] Hara K, Saito D, Shouno H. Analysis of function of rectified linear unit used in deep learning. In international joint conference on neural networks (IJCNN) 2015 (pp. 1-8). IEEE.

[41] Timothy Masters. Practical neural network recipes in C++. Morgan Kaufmann; 1993.

[42] Liodakis G, Arvanitis D, Vardiambasis IO. Neural network- based digital receiver for radio communications. WSEAS Transactions on Systems. 2004; 3(10):3308-13.

[43] Kapetanakis TN, Vardiambasis IO. Radiation performance of satellite reflector antennas using neural networks. In third international conference on mathematics and computers in sciences and in industry (MCSI) 2016 (pp. 85-8). IEEE.

[44] Konstantaras A, Varley MR, Vallianatos F, Collins G, Holifield P. Neuro-fuzzy prediction-based adaptive filtering applied to severely distorted magnetic field recordings. IEEE Geoscience and Remote Sensing Letters. 2006; 3(4):439-41.

[45] Konstantaras A, Varley MR, Vallianatos F, Makris JP, Collins G, Holifield P. Detection of weak seismoelectric signals upon the recordings of the electrotelluric field by means of neuro-fuzzy technology. IEEE Geoscience and Remote Sensing Letters. 2007; 4(1):161-5.

[46] Kapetanakis TN, Vardiambasis IO, Ioannidou MP, Maras A. Neural network modeling for the solution of the inverse loop antenna radiation problem. IEEE Transactions on Antennas and Propagation. 2018; 66(11):6283-90.

[47] Kapetanakis TN, Vardiambasis IO, Lourakis EI, Maras A. Applying neuro-fuzzy soft computing techniques to the circular loop antenna radiation problem. IEEE Antennas and Wireless Propagation Letters. 2018; 17(9):1673-6.

[48] Tang J, Alelyani S, Liu H. Feature selection for classification: a review. Data Classification: Algorithms and Applications. 2014.

[49] Srivastava N, Hinton G, Krizhevsky A, Sutskever I, Salakhutdinov R. Dropout: a simple way to prevent neural networks from overfitting. The Journal of Machine Learning Research. 2014; 15(1):1929-58.

[50] Konstantaras AJ. Classification of distinct seismic regions and regional temporal modelling of seismicity in the vicinity of the Hellenic seismic arc. IEEE Journal of Selected Topics in Applied Earth
Observations and Remote Sensing. 2012; 6(4):185763.

[51] Konstantaras A. Deep learning and parallel processing spatio-temporal clustering unveil new ionian distinct seismic zone. Informatics. 2020; 4(4):1-10.

[52] Wang Y, Li S, Song J. Threshold-based evolutionary magnitude estimation for an earthquake early warning system in the Sichuan-Yunnan region, China. Scientific Reports. 2020; 10(1):1-12.

[53] Sammut C, Webb GI. Encyclopedia of machine learning. Springer Science \& Business Media; 2011.

[54] Zubkov SI. The appearance times of earthquake precursors. Izv. Akad. Nauk SSSR Fiz. Zemli (Solid Earth). 1987; 5:87-91.

[55] Drakatos G, Latoussakis J. A catalog of aftershock sequences in Greece (1971-1997): their spatial and temporal characteristics. Journal of Seismology. 2001; 5(2):137-45.

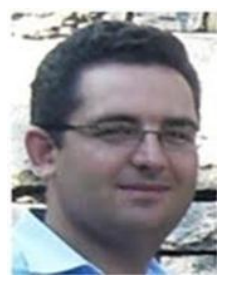

Dr. Antonios Konstantaras is an Associate Professor in Software Engineering in the Hellenic Mediterranean Univerity. He received his doctorate from the University of Central Lancashire, U.K. in 2004 Soft Computing. He has published over 25 journal articles in software engineering and serves as editor in 2 scientific journals.

Email: akonstantaras@hmu.gr

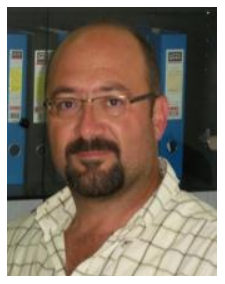

Nikolaos S. Petrakis is Lecturer at the Electronic Engineering Department of the Hellenic Mediterranean University, in Informatics with Specialization in Computer Programming and Computer Networks. He received the Diploma in Electrical Engineering (1990) from the Polytechnic Institute of Timisoara, and the Ph.D. degree in Reliability and Computer Engineering (1995) from the Technical University of Timisoara, Romania. He has authored or coauthored 18+ research conference papers. Dr. Eng. Nikolaos Petrakis is an active member of the Institute of Electrical \& Electronic Engineers (IEEE), Technical Chamber of Greece and Hellenic Association of Electrical Engineering.

Email: nik.s.petrakis@hmu.gr

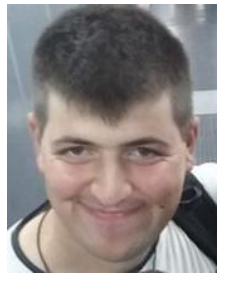

Frantzeskakis Theofanis is a $\mathrm{PhD}$ candidate in Electronic Engineering at Hellenic Mediterranean University (HMU). He received his Masters from Hellenic Mediterranean University (HMU) in 2020 in Telecommunication \& Automation Systems. He has published, 3 articles in Big Seismic Data and Parallel Programing. Email: tfaniz@yahoo.gr 


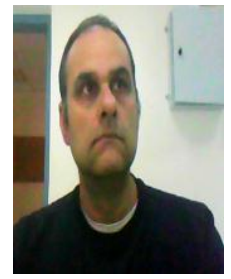

Emmanouil Markoulakis is a $\mathrm{PhD}$ candidate in Electronic Engineering at Hellenic Mediterranean University (HMU). He received his MPhil from Technical University of Crete (TUC) in 2011 in Electrical and Computer Engineering. He has published recently in the last 4 years, 5 articles in various fields of electrical engineering and physics.

Email: markoul@hmu.gr

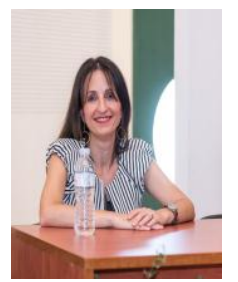

Katerina Kabassi is Associate Professor and Head of the Department of Environment at Ionian University. She received her $\mathrm{PhD}$ from University of Piraeus in 2004. She has published over 100 articles in international peer reviewed journals, books and conferences related to software engineering.

Email: kkabassi@ionio.gr

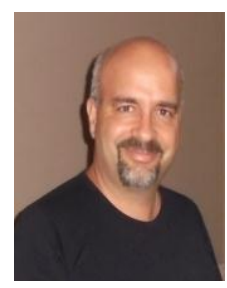

Ioannis O. Vardiambasis Ioannis Vardiambasis received the Diploma of Electrical Engineering and the $\mathrm{PhD}$ degree in Electrical \& Computer Engineering from the National Technical University of Athens (NTUA), Greece, in 1991 and 1996, respectively. $\mathrm{He}$ is currently an Associate Professor on "Satellite \& Microwave Communications" in the Department of Electronic Engineering of the Hellenic Mediterranean University (HMU). He has served in several administrative positions (Dean, Vice-Dean, Head of Department, Director of Division) at HMU for many years. He has published more than 100 scientific papers in referred journals and international conference proceedings.

Email: ivardia@hmu.gr

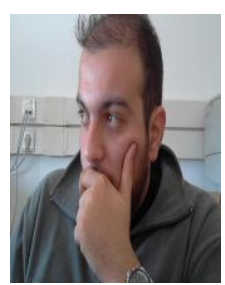

Theodoros Kapetanakis holds a B.S. degree in Electronic Engineering and an MSc in Telecommunication \& Automation Systems from the Hellenic Mediterranean University (HMU), also he holds a PhD from the Department of Informatics and Telecommunications, of University of Peloponnese (UoP). $\mathrm{He}$ is currently a postdoctoral researcher at the Department of Electronic Engineering at HMU and he has published more than 30 original papers in international peer review journals and conferences.

Email: todokape@hmu.gr

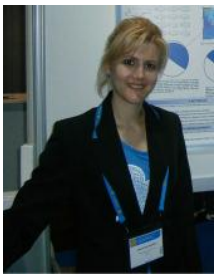

Alexandra Moshou Alexandra Moshou is a Dr. Seismologist and a post-doc researcher at Hellenic Mediterranean University. She received her doctorate from the National and Kapodistrian University of Athens in 2011 in Mathematical Methods in Seismology. She has published over 13 articles in various fields of seismology.

Email: amoshou@noa.gr

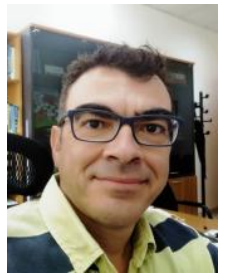

Emmanuel Maravelakis is an Associate Professor and the head of the Design, Manufacturing \& Automation Laboratory (DMA-Lab) of the Hellenic Mediterranean University. His main research interest area includes CAD/CAM systems, 3D Modeling and Reverse Engineering.

Email: marvel@hmu.gr

Appendix I

\begin{tabular}{lll}
\hline S. No. & Abbreviation & Description \\
\hline 1 & ${ }^{\circ}$ E & Degrees East \\
\hline 2 & ${ }^{\circ} \mathrm{N}$ & Degrees North \\
\hline 4 & 3D & Three-Dimensional \\
\hline 5 & CompuTech & $\begin{array}{l}\text { Institutionalized Laboratory of } \\
\text { Computer Technology, Informatics } \\
\text { \& Electronic Devices }\end{array}$ \\
\hline 6 & EQ & Earthquake \\
\hline 7 & GI-NOA & $\begin{array}{l}\text { Geological Institute of the National } \\
\text { Observatory of Athens }\end{array}$ \\
\hline 8 & Lat & Lattitude \\
\hline 10 & Long & Longitude \\
\hline 11 & Mag & Magnitude \\
\hline 12 & MMSR & Minute \\
\hline 13 & N/A & Monthly Mean Seismicity Rates \\
\hline 14 & ONEIROS & Mayleigh-based \\
\hline 15 & Sec & Not Applicable \\
\hline
\end{tabular}

